\title{
Chest $x$-ray: tips for the clinician
}

\begin{abstract}
The history of radiology began in 18th century with the experimental discovery of x-rays by a German physicist Wilhelm Conrad Roentgen, making it possible to evaluate structures inside the human body. Since then, the radiologic techniques and evaluation methods have improved greatly. The purpose of this paper is to propose a systematic evaluation method of chest radiographs to improve the quality of the evaluation by the physician that is attending the patient or radiologist in training, we will use normal and pathological cases to illustrate and improve the learning of our proposed interpretation method.
\end{abstract}

Keywords: chest $\mathrm{x}$-ray, physician evaluation, systematic chest $\mathrm{x}$-ray interpretation
Volume 6 Issue 5 - 2019

Paulo Cezar Leal Ecclissato,' Diego Jordão

Lino Dias, ${ }^{2}$ Amanda Selvátici dos Santos

Dias, ${ }^{2}$ Jorge Henrique Safady,' Lucas

Figueiredo Maia Roque,' Erika Suemi Obata,' Luiza Costa Nelli'

'Department of Radiology, Faculty of Medicine of Marilia, FAMEMA, Brazil

${ }^{2}$ Santos Dias Medical Care, Brazil

Correspondence: Diego Jordão Lino Dias, Santos Dias Medical Care, 140 Imbituba Street, 185 apartment, São Paulo-SP, 03।32-090, Brazil, Tel 55 (14) 991613327,

Email diegojorda@msn.com

\section{Introduction}

Chest radiography has been widely used in medicine since the discovery of X-ray by Wilhelm Roentgen in $1895 .{ }^{1}$ Nowadays the importance of performing a good clinical evaluation, added to the systematic interpretation of radiographs has gained space. ${ }^{2-4}$ In Brazil, especially in the Faculty of Medicine of Marilia - FAMEMA, Dr. Paulo Cezar Leal Ecclissato teaches for several years the method we will call FAMEMA-ECCLISSATO. Methodology based on the analysis of structures from the periphery to the center that will be detailed in this article with figures and drawings. This method has been taught to residents of Radiology and Diagnostic Imaging over the years, making the evaluation of chest radiographs more complete and faster. It's a way to "indoctrinate" the interpreter to use the same steps to evaluate all chest $\mathrm{x}$-rays equally and wholly. As a basis for interpreting this article, we would use digital chest X-ray, with a patient about 1.8 meters from the Chassis. Ideal inspiration, positioning and image penetration with posteroanterior (PA) and lateral (Lat) radiographs. ${ }^{5}$

\section{Famema-Ecclissato method}

The FAMEMA-ECCLISSATO systematic approach to the chest x-ray consists in an evaluation of the human anatomic structures that starts with a "periphery to center" fashion, divided in 6 steps (Figure 1-10).

\section{Step I}

\section{"Soft tissue": pay attention to the skin and subcutaneous tissue of the patient}

Start the x-ray evaluation by looking at the skin and subcutaneous tissue and look for findings like muscular hypotrophy/hypertrophy, subcutaneous emphysema (common in traumatized patients), calcifications (Common in cysticercosis), solid lesions or masses (Lymphatic masses, tumor-like lesions), etc (Figure 1).

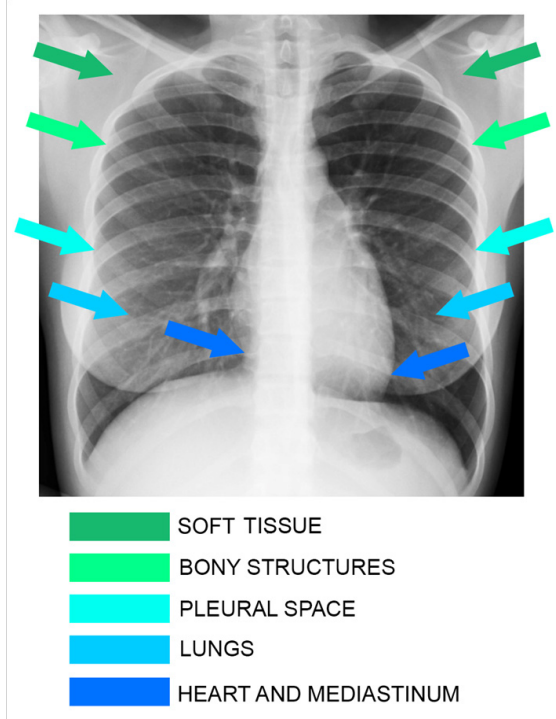

Figure I Step I to 5.Analysis the chest x-ray from the periphery to the center. The arrows indicate the structures to be evaluated on chest radiography.

\section{Step 2}

\section{"Bony structures": look closely and individually to the patient's ribs, clavicles, sternum and scapula}

Consider the dimensions, density and margins of the ribs, clavicles, scapula, thoracic spine and sternum. Look for lytic (radiolucent) or blastic (radiodense) lesions, fractures, degenerative disease and anatomical variants, etc. To evaluate the thoracic spine, lateral view gives us better information as the cardiac silhouette overlaps the spine at PA view (Figure 2). 


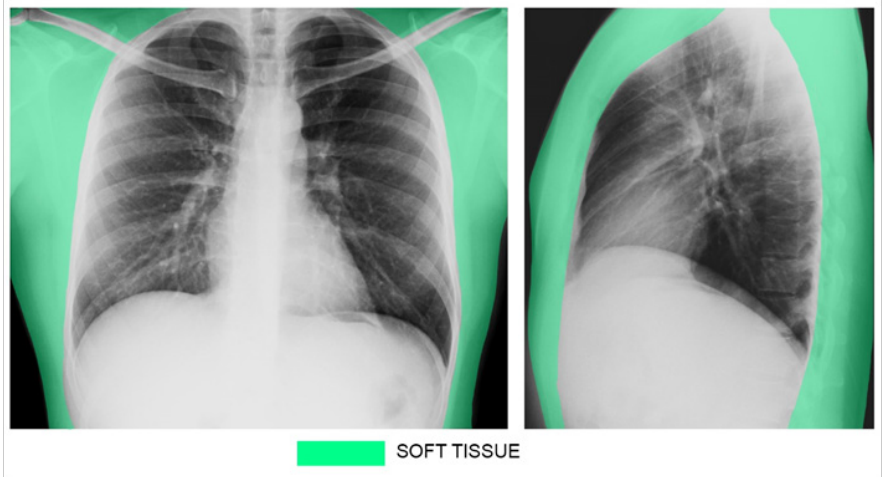

Figure 2 The green painted area signals the soft tissues to be evaluated at step I.

\section{Step 3}

"Pleural space": evaluate the visceral and parietal pleurae, diaphragm cupules and the upper abdomen (Liver, gastric bubble, spleen and intestines, if possible)

Start with the pleurae, then the diaphragm cupules and finally the upper abdomen. Look for pleural thickening (effusion and calcifications), costophrenic angle blunting (effusion, atelectasis, etc.), upper abdomen (pneumoperitoneum, ascites, "Stack of coins" sign) (Figure 3).

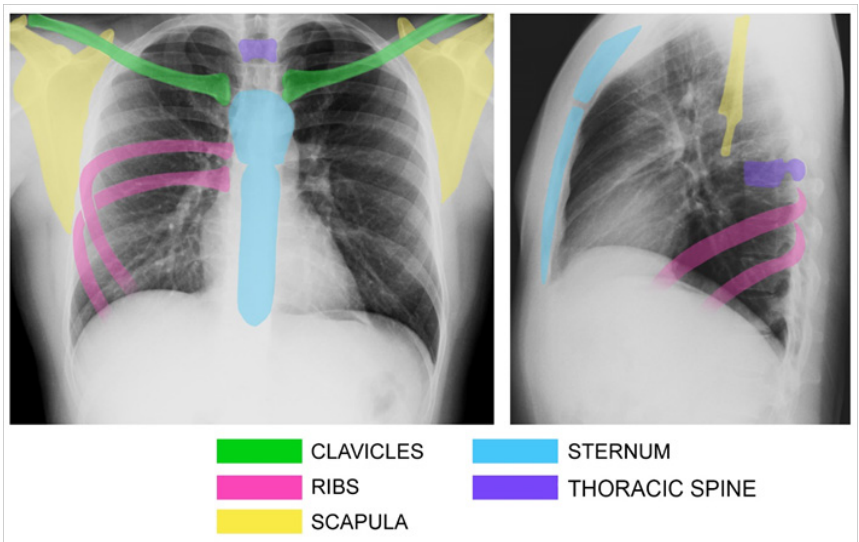

Figure 3 Bony structures to be evaluated at step 2 .

\section{Step 4}

\section{"Lungs": Evaluate each lung individually paying attention to its parenchyma, hilum and tracheobronchial tree}

Look for opacities, air bronchogram (common in bronchopneumonia), nodular and hilar lesions (suggestive of lymphadenomegaly or neoplasms), cavitation (may happen in tuberculosis, pulmonary abscess, pneumatoceles), radiolucent lesions (emphysema and blebs), reticular opacities (interstitial diseases like in congestive heart failure will show Kerley's lines), pneumothorax, etc. At this step it is necessary to evaluate the inspiration, in a welldone radiograph; you need to count between 5 and 6 anterior ribs above the right hemidiaphragm. If it is below the 6th anterior rib, it indicates hyperinsufflation, and if it is upper than 5th anterior rib it demonstrates hypoinsufflation (Figure 4-7).

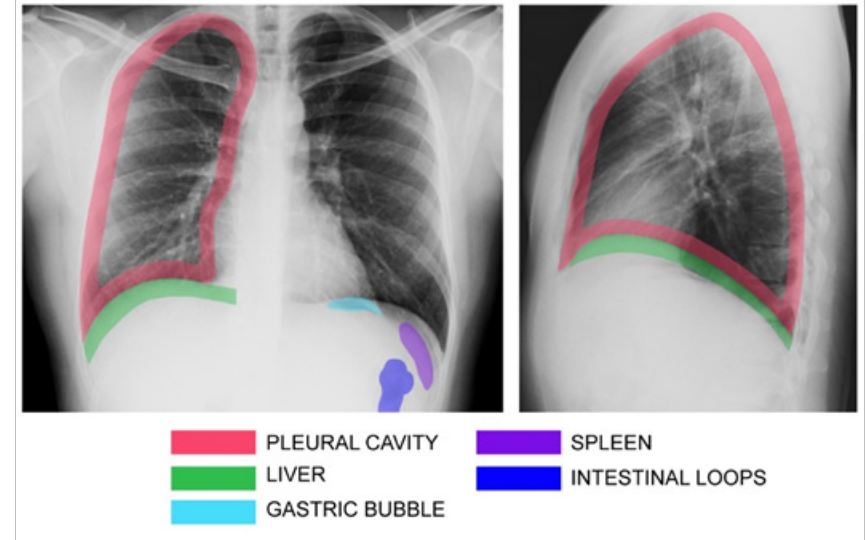

Figure 4 In red color, pleural space at PA and lateral incidences painted in red.

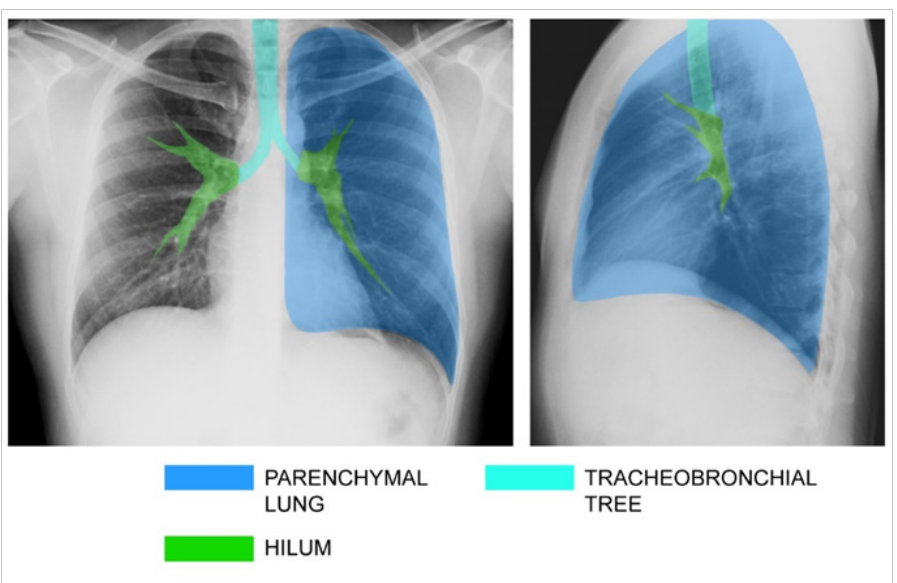

Figure 5 In blue, the lung parenchyma, hilum and tracheobronchial tree to be evaluated at step 4.

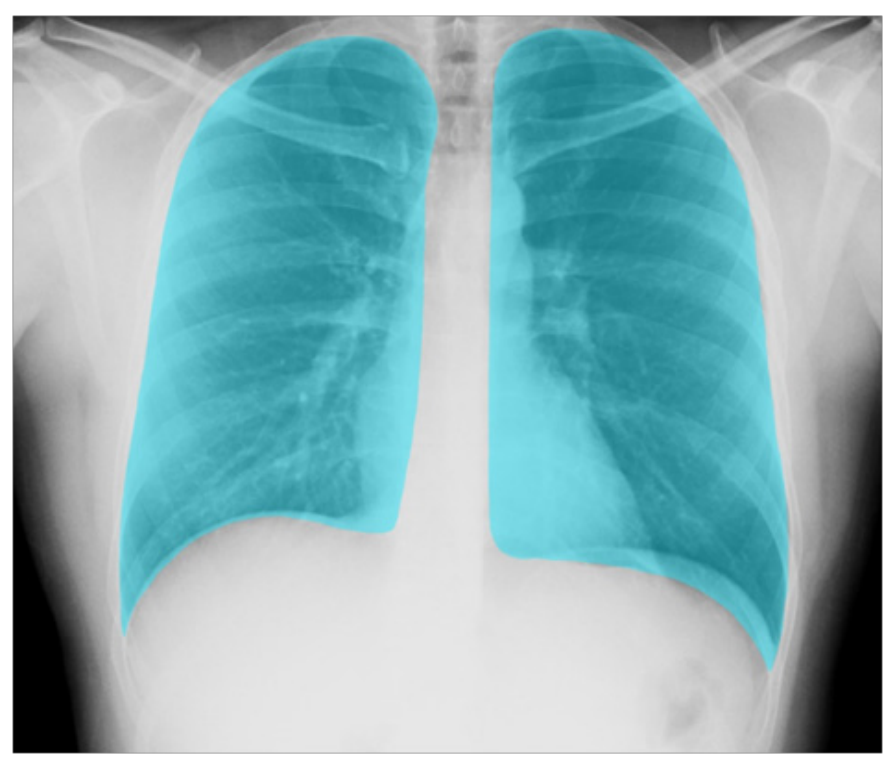

Figure 6 In light green, the symmetry of the lung parenchyma. Look for alveolar or reticular opacities. 

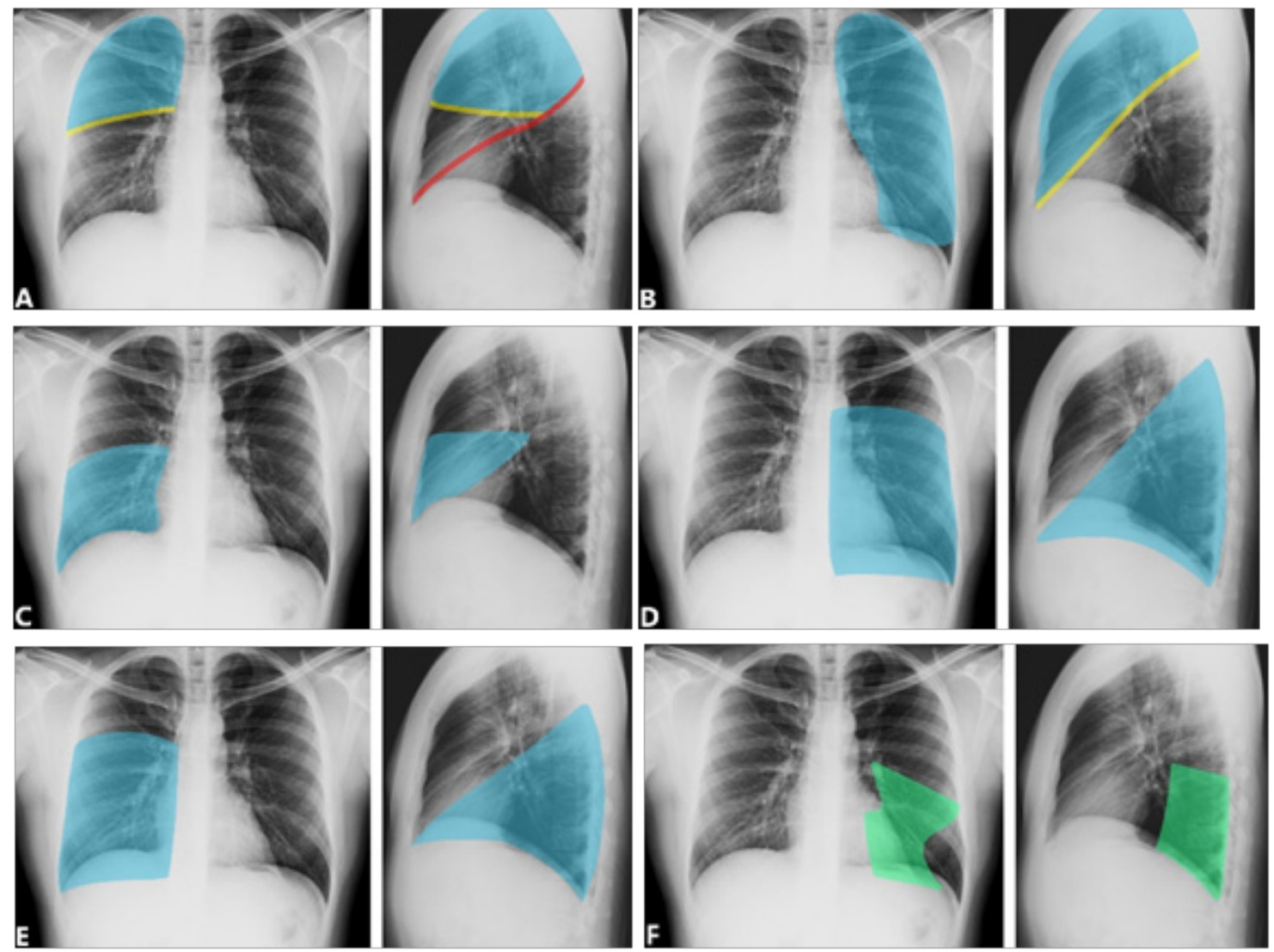

Figure 7 Lobar division of the lung. (A) Right oblique fissure (red line), separating the right lower lobe from the upper and middle lobe. Horizontal fissure (yellow line) separating the upper lobe from the middle lobe. (B) Left oblique fissure (yellow line) separating the upper lobe from the left lower lobe. (C) Medium lobe. (D) Lower left lobe. (E) Right lower lobe. (F) Projection of the posterior segment of the left lower lobe.

*Note:At X-ray we recommend dividing the lung anatomy by lobes, leaving the lobar anatomical segmentation for computed tomography.

\section{Step 5}

"Heart and mediastinum": is the heart enlarged? is there any mediastinal mass?

Observe the dimensions and contours of the mediastinum (cardiac size and mediastinal vascular structures), mediastinal subdivisions (anterior, middle, and posterior). Look for Cardiomegaly, vascular enlargement (e.g.: Pulmonary hypertension), mediastinal masses (Lymphoma, neoplasms, etc.), diaphragm hernias, megaesophagus, etc. It is necessary to estimate the cardiothoracic ratio $(\mathrm{CTR})^{7}$ We consider as cardiomegaly when CTR is $\geq 0.5$ (Figure 8).

Mediastinum: Anterior (prevascular), middle (visceral) and posterior (paravertebral) by ITMIG Classification. ${ }^{6}$ In the following figures we present a radiograph with the regions dividing the thorax into 3 parts and a drawing with the anatomical landmarks (Figure 9).

\section{Step 6}

"Panoramic View": look to the chest X-Ray as a whole (Big Picture Mode), is there any asymmetry? is some structure stands out?

The last step it is necessary to see the whole radiography. Observe if nothing went unnoticed. Finally, an example of Normal Chest $\mathrm{X}$-Ray, this is the pattern of normality in a PA and lateral radiograph that we should keep in mind, well-inspired, adequate penetration and aligned (Figure 10).

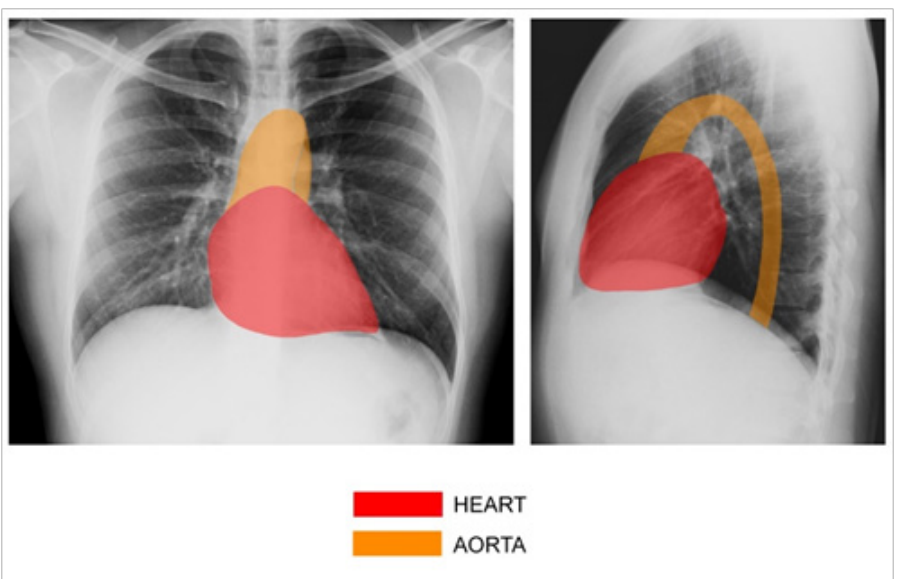

Figure 8 Heart and Mediastinum are signalized. 

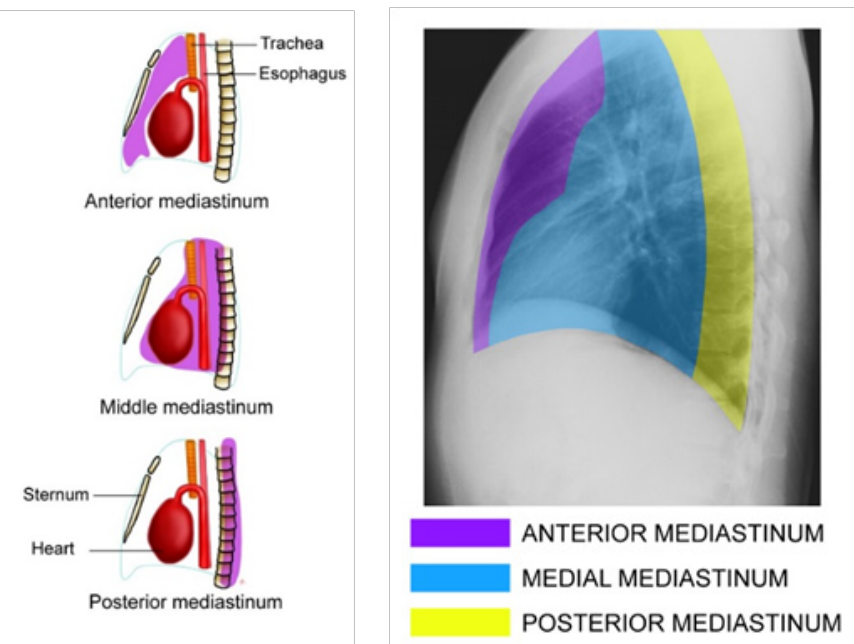

Figure 9 On the right we have a drawing of the structures of mediastinum by ITMIG Classification. On the left, a radiography with this division.

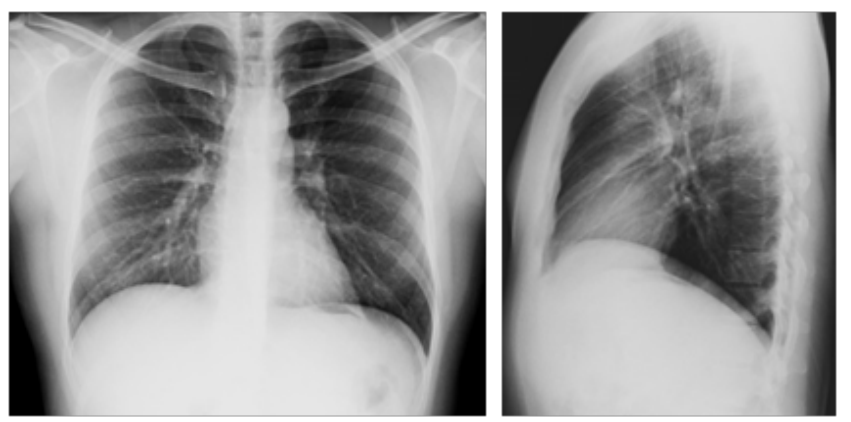

Figure 10 Normal Chest X-Ray on PA incidence (left) and lateral incidence (right).

\section{Clinical discussion and pathologies}

The chest radiography is an extremely important complementary exam to help the clinician on their medical routine, either in the emergency room or at the ambulatory. There are a huge spectral of use this exam in our medical routine, probably because the chest x-ray is a cheap, easy access and very helpful exam. When we indicated to the patient to do a chest $\mathrm{x}$-ray, we must have in mind some aspects about the exam and their interpretation and it will help us on taking important clinical decisions. We will see in this article some of the applications of $\mathrm{x}$-ray, both in emergency room and ambulatorial routine. At this part of the evaluation we will look for every change on muscles, skin, subcutaneous and some devices that the patient may have using, like chest drain (Figure 11), nasogastric catheter or cardiac monitoring electrodes.

In malnourished patients for example, we may find some changes like muscle atrophy and decrease of subcutaneous tissue. We also can find breast asymmetry present in some types of breast cancer or previous mastectomy. In trauma context the evaluation may demonstrate injuries like subcutaneous emphysema (Figure 11).

Bone fractures, fracture healing and degenerative process (Figure 12) can be seen in the evaluation of the bone framework. In patients with decompensated heart failure commonly we will see costophrenic sinuses erased bilaterally. Pneumonia and some paraneoplasic syndromes can also course with pleural effusion (Figure 13). In a context of trauma or perforation of hollow viscera, we may encounter pneumoperitoneum (Figure 14). In the evaluation of lung parenchyma, we will see signal that corroborate with the diagnosis of pneumonia, tuberculosis, chronic obstructive pulmonary disease (Figure 15) or even findings like solitary pulmonary nodule that must be investigated, mainly in patients that have risk factors to lung cancer. Evaluating a patient who has high blood pressure, it is important measure if their disease is already causing cardiac remodeling as cardiomegaly (Figure 16) or if there are aortic involvement when echocardiography is not available. The chest X-ray could help us measure the CTR (Figure 17). In cases of pericarditis there may be an increased cardiothoracic ratio with a globular P outline if there is co-existing pericardial effusion. Finally, we believe in the simplicity and practicality of our evaluation method. Being a form of clinical-radiological systematization, making the FAMEMA-ECCLISSATO method of interpreting chest radiography very useful in daily practice. ${ }^{7}$

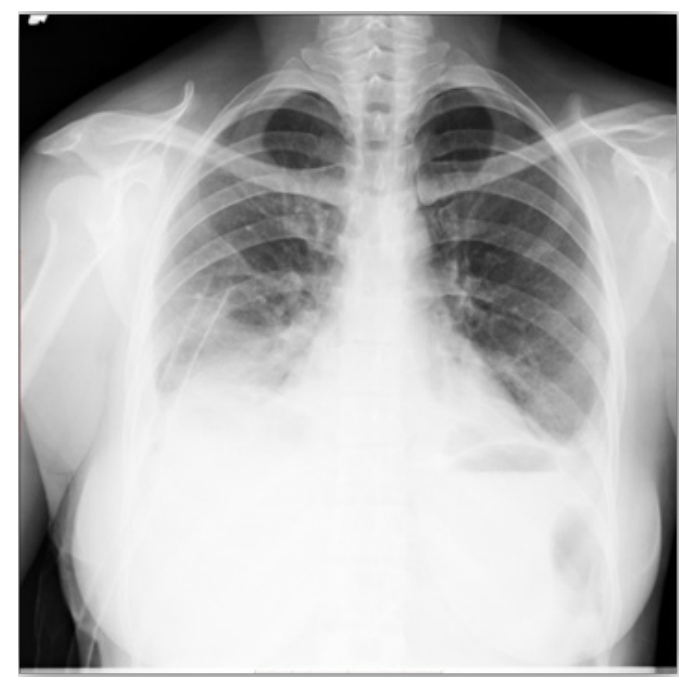

Figure II Right chest drains, associated with soft tissue enlargement in the right thoracic base, with slight subcutaneous emphysema, all evaluated at step I.

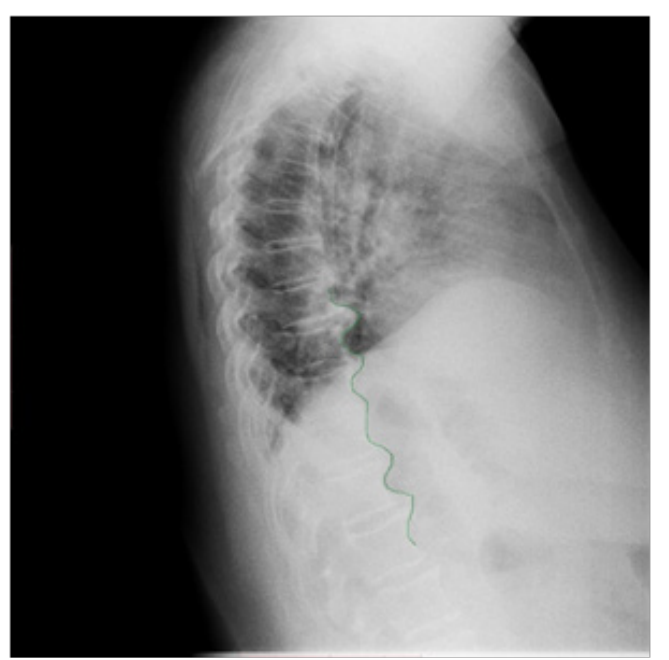

Figure 12 Example of bone changes: anterior longitudinal ligament calcification (green line), look for this at step 2. 


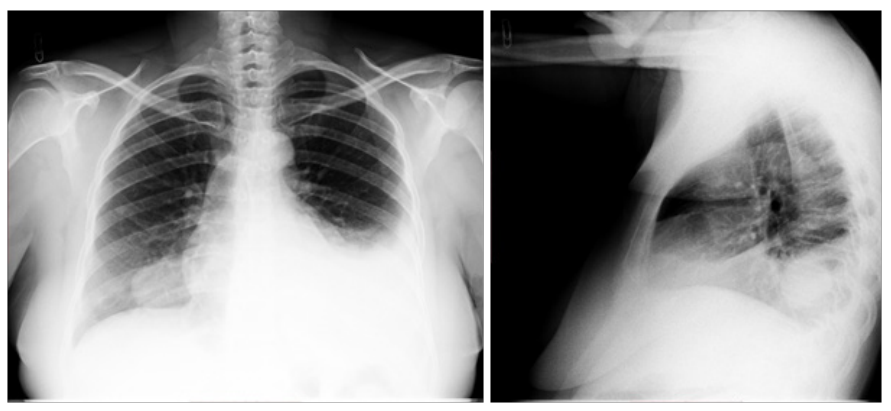

Figure I 3 Findings at step 3: Lung mass in right lower lobe and left pleural effusion, evidenced in both incidences (PA and lateral).

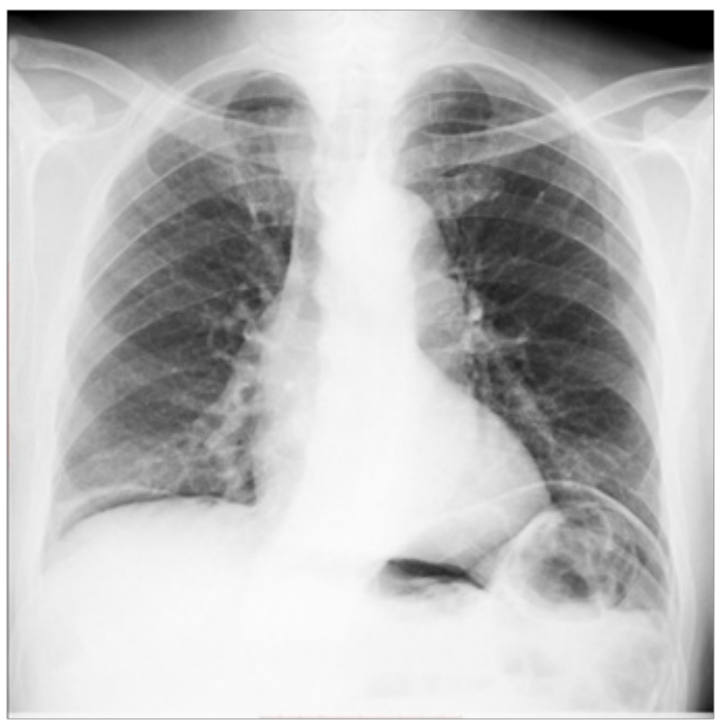

Figure 14 Pneumoperitoneum - Example of findings at step 3 evaluation (diaphragm cupules and the upper abdomen).

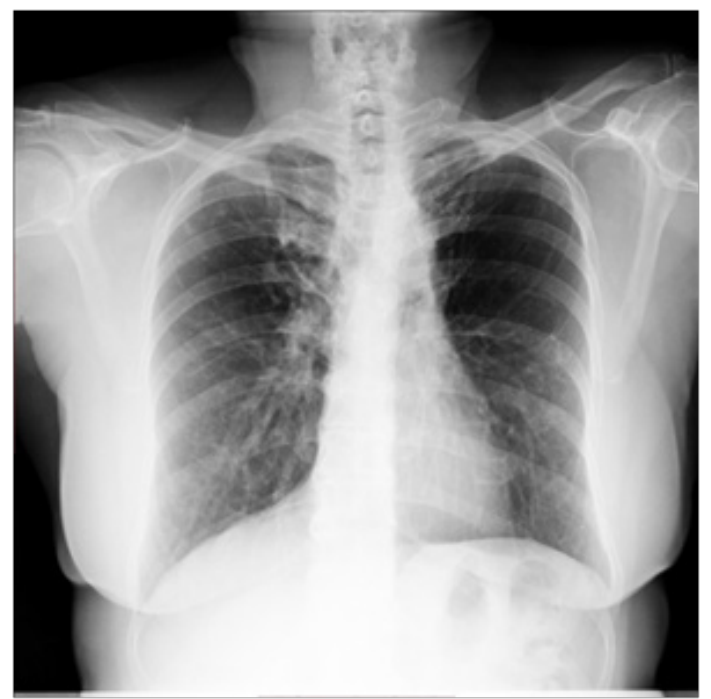

Figure I 5 Example of findings of lung parenchyma, hilum and tracheobronchial tree: Patient with chronic obstructive pulmonary disease (COPD) showing Bronchiectasis and cephalization of vasculature.

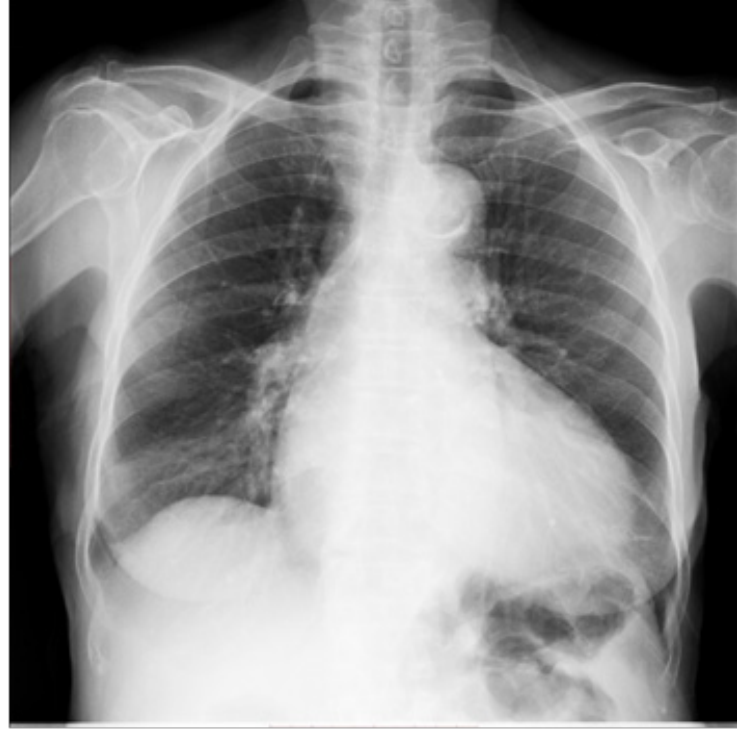

Figure 16 Patient with congestive heart failure showing cardiomegaly and long aorta with parietal calcifications.

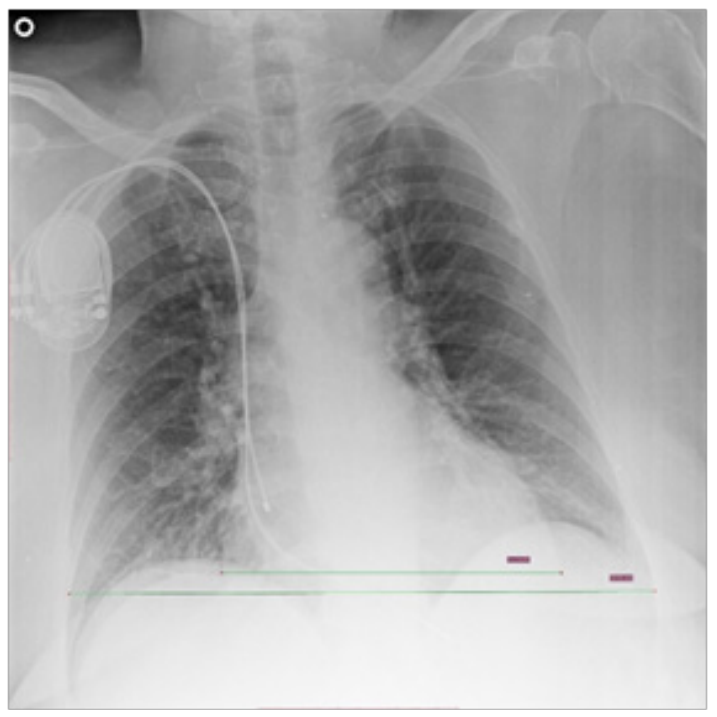

Figure 17 Another patient with pacemaker, congestive heart failure and cardiomegaly. Showing practical method of estimating CTR.

\section{Acknowledgments}

None.

\section{Funding}

Self funding.

\section{Conflicts of interest}

The author declares that there is no conflict of interest.

\section{References}

1. Panchbhai AS. Wilhelm Conrad Röntgen and the discovery of X-rays: Revisited after centennial. J Indian Acad Oral Med Radiol. 2015;27(1):90-95. 
2. Duong M, Timoney PJ, Macnicholas R, et al. ABC's of Chest X-Rays. TSMJ Clinical Medicine. 2001;2:11-14.

3. Talley Nicholas Joseph, Simon O'Connor. Clinical Examination: A Systematic Guide to Physical Diagnosis. Oxford: Blackwell Science; 1996.

4. Watters Jennifer M. A systematic approach to basic chest radiograph interpretation: a cardiovascular focus. Can $J$ Cardiovasc Nurs. 2014;24(2):4-10.

5. Moffett BK, Panchabhai TS, Nakamatsu R, et al. Comparing posteroanterior with lateral and anteroposterior chest radiography in the initial detection of parapneumonic effusions. Am J Emerg Med. 2016;34(12):2402-2407.

6. Carter BW, Benveniste MF, Madan R, et al. ITMIG Classification of Mediastinal Compartments and Multidisciplinary Approach to Mediastinal Masses. RadioGraphics. 2017;37(2):413-436.

7. S Zaman MJ, Sanders J, Crook AM, et al. Cardiothoracic ratio within the "normal" range independently predicts mortality in patients undergoing coronary angiography. Heart. 2007;93(4):491-494. 